\title{
Dialogue entre la recherche anthropologique et l'exploration artistique
}

\section{Dialogue between anthropological research and artistic exploration}

\author{
Célia Riboulet ${ }^{1}$ \\ ${ }^{1}$ LARA-SEPPIA, Université Toulouse Jean-Jaurès
}

\begin{abstract}
RÉSUMÉ. Cet article ouvre une réflexion sur les enjeux de la rencontre entre l'art et l'anthropologie et conjointement sur les relations qui se nouent entre la fiction et le documentaire. La réflexion s'initie dans une expérience concrète de l'ailleurs qui invite à repenser les limites de la construction des mondes, comme celles des disciplines. Deux œuvres étudiées problématisent cette rencontre : La mangeuse de pierres (2019) de Célia Riboulet et Juegos de Herencia (2011) de Clemencia Echeverri.

ABSTRACT. This paper opens a reflection on the stakes of the encounter between art and anthropology and jointly on the relations between fiction and documentary. Reflection is initiated in a concrete experience from elsewhere that invites us to rethink the limits of the construction of worlds, such as those of disciplines. Two works studied problematize this meeting: La mangeuse de pierres (2019) by Célia Riboulet and Juegos de Herencia (2011) by Clemencia Echeverri.

MOTS-CLÉS. art, fiction, anthropologie, documentaire.

KEYWORDS. art, fiction, anthropology, documentary.
\end{abstract}

L'eau s'engouffre dans son corps comme dans le lit d'une rivière. Elle est épaisse, humide. Elle caresse les parois de sa bouche, poursuit son chemin vers le dedans. Il entend le bruit de l'eau, sous la pierre, sous la terre. Son visage contre le sol perçoit la froideur; au loin, le bruit de l'eau sous les roches, le battement de son flot. Ses mains soulèvent la terre, elles grattent les cailloux, pour creuser un tunnel, vers le cœur de la montagne, pour s'approcher d'elle, pour l'entendre palpiter, grogner, pour l'écouter vivre. Sa peau sur la roche se frotte, elle cherche la chaleur de la pierre. Son corps se niche entre les plis d'une paroi, son souffle se mêle à l'air, son corps se roule dans la pierre. L'air qui effleure la roche est le même que celui qu'il inspire. Sa peau s'affine, se désépaissit. Elle devient poreuse. Elle absorbe l'air, la pierre, le soleil, l'eau de la terre. La vie le traverse, comme elle traverse ce qui l'abrite, ce qui le couve. Il dépose son être dans les plis de ces montagnes, là où l'eau, le ciel, la terre et l'air communiquent. Dans le silence du soi, il a fait résonner les gouttes de pluie, les rayons de lumière, l'épaisseur du souffle; il s'est nourri de la vie en dehors de lui.

La différence entre le paysage et l'environnement est une question de différence de point de vue. Vit-on à l'intérieur d'un monde ou bien vit-on à l'extérieur du monde ? Est-on affecté par son monde ou bien vit-on isolé du monde? L'environnement n'est pas ce décor immuable où se déroulent les activités humaines, il ne cesse de se renouveler au cours du temps et de nos vies. Nous façonnant autant que nous-mêmes le façonnons. C'est cette distinction que l'anthropologue anglais Tim Ingold pointe du doigt quand il sépare la «réalité de » et la « réalité pour». Dans la « réalité de », le monde physique des objets neutres n'est visible qu'à l'observateur détaché et extérieur. Dans la « réalité pour », le monde est constitué en relation à l'organisme ou à la personne dont il est l'environnement. Comment alors l'environnement peut-il affecter, toucher le corps ? Comment inviter le corps à vivre une expérience complète qui le mette en relation avec son environnement ? Jean-Marc Besse, directeur de recherche au CNRS de l'équipe EHGO/UMR Géographies-cités, co- 
directeur de la rédaction de la revue Les Carnets du paysage, reconnaît dans la marche une des voies qui pourrait mener à cette expérience ; et plus précisément le moment de fatigue dans la marche qui pourrait restituer au corps sa porosité ${ }^{1}$. Cette notion de porosité me semble très intéressante, parce qu'elle figure une image très révélatrice : le corps n'est plus limité par une couche imperméable à l'environnement, il est au contraire poreux, dans le sens où il peut s'imprégner d'éléments extérieurs à lui-même, il est immergé dans son environnement.

Comment cette immersion est-elle possible ou à peine envisageable ? Jean-Marc Besse suppose qu'il est sans doute nécessaire de sortir des codes occidentaux de la pensée paysagère pour accéder à ce qu'il nomme l'évènement-paysage. Il s'agit sans doute dans cette prise de distance d'apprendre à désapprendre, d'apprendre à défaire les filtres qui nous éloignent du monde et de mettre en avant notre corps et notre expérience dans la saisie de ce qui nous entoure. Jean-Marc Besse propose de se déplacer vers la Chine, je me suis déplacée pour ma part au Mexique. L'expérience mexicaine me permit de vivre l'évènement-environnement en bousculant la perméabilité des frontières entre l'homme et ce qui l'entoure, en désobéissant aux divers prédicats qui me liaient au monde, « prédicat-monde $»^{2}$ qui éclaire le cosmos sous un certain jour (et qui à ces marges naît comme autre chose).

\section{La mangeuse de pierre}

Dans la série photographique que j'ai réalisée en 2019 avec l'aide de Sébastien Cassin, $L a$ mangeuse de pierre, une assiette remplie de pierre est servie devant une femme. La scénographie de l'image plante un décor quelque peu surréaliste. La table est installée dans un environnement montagneux. Les pierres sont ingérées petit à petit. Au fur et à mesure de l'ingestion, le corps se teinte d'une couleur proche de la pierre en partant des pieds. Par strates, la couleur de la pierre s'élève jusqu'à recouvrir tout le corps. Une fois que le corps est entièrement recouvert de cendre figurant la pierre - s'extraient de l'intérieur du corps, les organes. Les organes sont déposés dans l'assiette. La dernière image qui clôt la série présente ces organes installés sur une pierre, comme pourrait l'être un cairn balisant un chemin de montagne.

Cette série s'est construite à partir d'une réflexion sur la question de la perméabilité des frontières. C'est l'homme dans son état social et culturel qui est interrogé. La table, l'assiette, les vêtements, les gestes, le paysage, aussi sont ceux de l'homme occidental. Il n'y a pas de sauvagerie dans ces images, tout est dans la retenue. Cette femme mangeuse de pierres donne une forme visible et sensible à mon expérience. Cette série ne relate pas un rituel existant, elle ne documente pas des faits réels, elle est cependant inspirée de différents faits réels, mythes et histoires issus de l'ontologie mexicaine. La cendre qui recouvre la peau et le vêtement donne au corps humain une qualité différente. Elle le recouvre, tout en laissant transparaître le corps et le vêtement. C'est la combinaison des qualités du corps alliées aux qualités de la cendre qui donne le caractère hybride de cette femme-pierre. Ce n'est ni une femme, ni une pierre, c'est une femme qui a intégré les qualités

1 BESSE Jean-Marc, op.cit., p. 51, cite BOUVIER Nicolas, Les chemins du Halla-san, dans Journal d'Aran et d'autres lieux, Paris, Payot, 2001, p. 126 : « La fatigue de la marche, écrit Nicolas Bouvier au seuil du monastère Hae-in-sa, en Corée, rend poreux, ouvert au langage d'un lieu : impossible de franchir ce parvis sans se sentir allégé, lavé de quelque chose. "

2 Berque Augustin, De terre en monde / La poétique de l'écoumène, dans Berque Augustin, BIASE (de) Alessia, BonNIN Philippe (dir.), L'habiter dans sa poétique première, Actes du colloque de Cerisy-la-Salle, Paris, Éditions donner lieu, 2008, p. 245. 
de la pierre, sa matière, sa mémoire, son temps. La pierre, la chair, le corps humain, entrent en dialogue. C'est dans le dialogue des matières et des formes que se tissent des fictions. C'est dans leur mise en contact qu'ont lieu les transformations. En ouvrant les frontières et les limites des formes et des matières, elles peuvent ainsi entrer en relation pour ouvrir le domaine de la signification et du sens. 

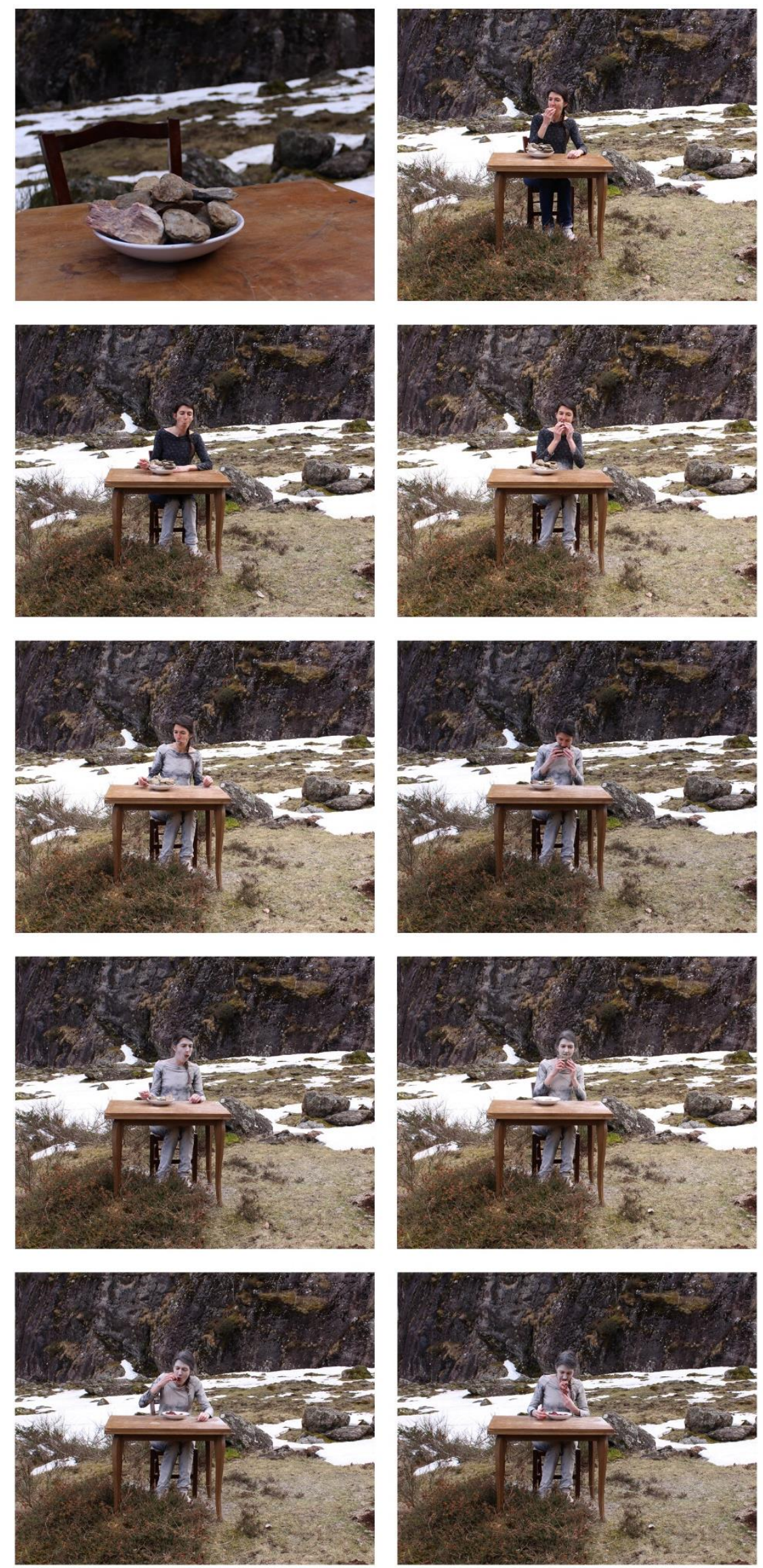
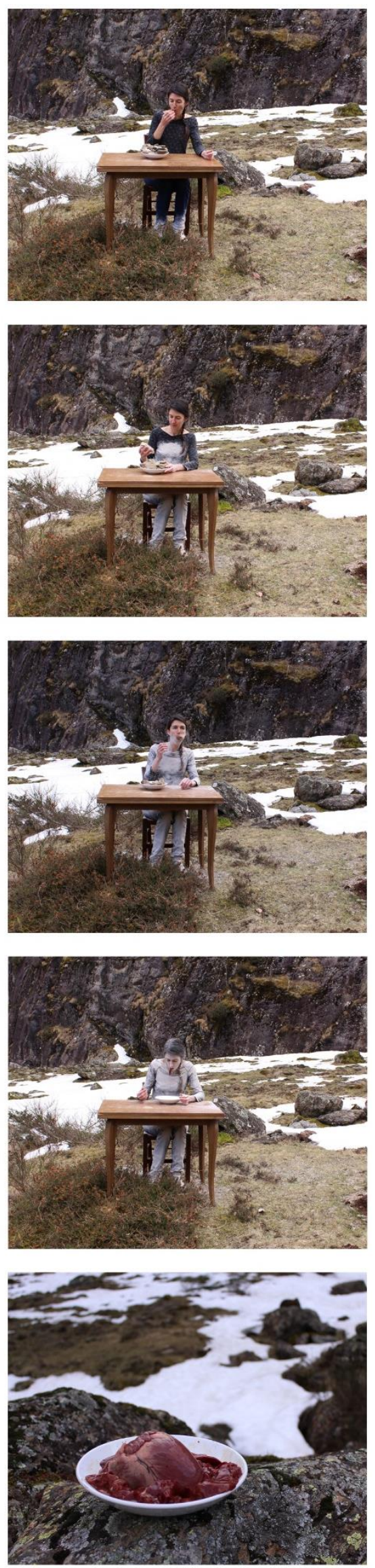

Riboulet Célia, La mangeuse de pierre, (photographies : Sébastien Cassin), série de 15 photographies, $16 \times 13 \mathrm{~cm}, 2019$. 

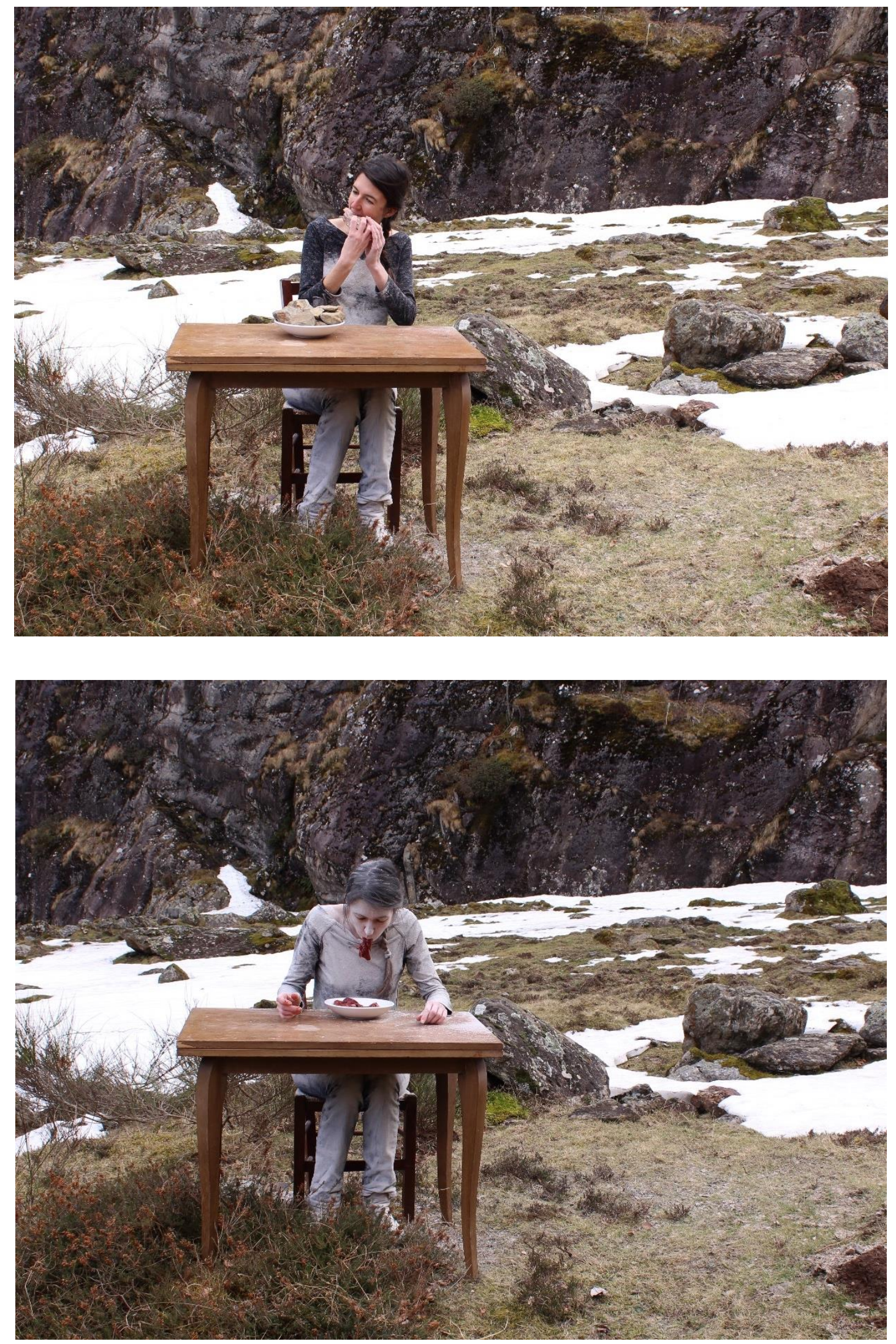

Riboulet Célia, La mangeuse de pierres, (Photographie : Sébastien Cassin), détails de la série, $16 \times 13 \mathrm{~cm}, 2019$. 
Se cristallise dans cette série un des enjeux théoriques de ma recherche, le déplacement du concept de paysage vers celui d'environnement. Ces images rendent sensible ce changement de point de vue. Le paysage installe l'homme face à la nature alors que l'environnement place l'homme dans son milieu. La mangeuse de pierre n'est pas dans un paysage, elle est dans un environnementmatière. Cette femme ingère un des composants de son environnement, la pierre. Métaphoriquement, elle déplace l'environnement de l'extérieur vers l'intérieur de son corps. Le paysage n'est plus face à elle, il est dans elle, c'est ainsi qu'il se transforme en environnement, son milieu. Ce fragment du milieu que la femme ingère, se mêle à elle, la modifie ; le changement de couleur extérieur traduit cette imprégnation. Elle ne se pétrifie pas, elle se transforme. Ensuite, le «vomissement » de ses organes peut être lu comme un don. Un don, entendu dans sa forme figurée, à savoir l'« action de (s') abandonner, de (se) remettre aux soins de quelqu'un, en se fiant à lui » ${ }^{3}$. Une fois que le corps est entièrement recouvert de cendre - figurant la pierre - s'extraient de l'intérieur du corps, les organes qui sont déposés dans l'assiette. C'est cela que réalise la mangeuse de pierres en donnant ses organes, elle se remet aux soins de son milieu, elle s'y livre, elle se fie à lui. Son action pourrait être traduite ainsi : le milieu fait partie d'elle, elle fait partie de lui, il vit en elle comme elle vit en lui.

Les frontières qui séparent les corps, les concepts, les matières, les cultures se dissolvent; l'histoire fictionnelle de la mangeuse de pierres déborde des cadres de nos constructions mentales, de nos possibles. Elle s'ancre dans le réel tout en le débordant. Elle tisse de nouvelles relations entre le réel, les mythes, les rites, l'histoire, l'autre, le milieu, le corps. Elle s'inscrit dans un dialogue entre la recherche anthropologique, l'exploration artistique et les mythologies personnelles. S'articule dans cette œuvre une étroite relation entre d'une part la démarche anthropologique et d'autre part la démarche artistique. 


\section{Entre l'art et l'anthropologie}

Pourtant, la recherche anthropologique et la recherche artistique ne sont pas investies des mêmes enjeux. Le théoricien de l'art Jean-Claude Moineau, dans un article intitulé L'artiste et ses «modèles " ${ }^{4}$, analyse la référence extra-artistique de l'art aux sciences et plus particulièrement à l'anthropologie. Pour l'auteur, l'apparition du paradigme de l'artiste comme anthropologue est à rapporter à l'entrée de l'art dans le champ élargi de la culture que l'anthropologie est traditionnellement supposée contrôler. Cette tendance s'inscrit dans des travaux d'artistes qui présentent et questionnent le problème des identités, de l' «autre culturel» dans une époque de globalisation. Les artistes réalisent des enquêtes de terrain au sein de leur quotidien, leur famille, leurs proches mais aussi à l'extérieur, dans les non-lieux, les terrains vagues, les friches, pour s'approcher des exclus, des «sans domicile fixe », des nomades, des migrants, des rejetés du système, des sans-papiers. Jean-Claude Moineau cite différents artistes. Entre autres, il mentionne le travail d'Anthony Hernandez qui photographie les « abris » des sans-abris de Los Angeles, sans jamais montrer les sans-abris eux-mêmes. Il évoque l'œuvre de Gabriela de Gusmao Pereira, Invention Street, qui expose des photographies de la débrouillardise, de l'inventivité, de l'art des SDF dans les rues de Rio de Janeiro au Brésil. Il cite aussi le travail de Jacqueline Salmon, Chambres précaires, qui présente des photographies des chambres d'accueil mises à la disposition des SDF pendant les mois d'hiver par les institutions caritatives. Ainsi que l'œuvre d'Andres Serrano qui dans sa série intitulée Nomades a photographié des SDF dans un studio improvisé au sein du métro new-yorkais.

Les photographies des différents artistes présentés ne tendent pas tant à dénoncer une certaine «misère » sociale et culturelle, qu'à réhabiliter des non-lieux oubliés, à porter le regard vers les marges, vers ce que l'on ne voit pas. Dans ces travaux, l'artiste devient anthropologue en établissant son terrain de recherche et d'action dans des espaces auparavant « réservés » aux anthropologues ou à ceux qui les occupent. C'est donc essentiellement la déterritorialisation et l'immersion dans un milieu distinct de l'atelier de l'artiste qui fondent les bases de la rencontre de ces deux domaines : l'art et l'anthropologie. Toutefois, l'artiste ne peut se confondre avec l'anthropologue, en ceci que de nombreux artistes n'ont pas directement affaire aux populations concernées, pas plus qu'ils n'agissent à produire de la science (même s'ils produisent parfois des savoirs). De nombreuses photographies magnifient, esthétisent les non-lieux et leurs habitants, sans pour autant montrer la relation de l'artiste avec ceux-ci. Cette esthétisation de la souffrance, de la misère peut d'ailleurs dans certains cas être dérangeante sur le plan de l'éthique. L'artiste n'en a pas non plus l'obligation ; rien ne lui dicte sa conduite ou le processus de création de ses images. Il est libre de déposer son regard et son appareil vers ce qui l'attire sans en donner une justification anthropologique. L'artiste et l'anthropologue partagent des thèmes, des terrains de recherches, cependant leurs méthodes d'analyse et leurs productions sont totalement différentes. 


\section{Entre la fiction et le documentaire}

Une idée courante veut que le documentaire parte de quelque chose qui existe tandis que la fiction ferait exister ce qui n'existe pas. Dans le domaine de l'art, les artistes ont toutefois tendance à ne pas marquer si clairement la distance entre ce qui relève de la réalité et ce qui relève de la fiction. La relation réalité-fiction-documentaire constitue une problématique féconde au cœur de nombreuses œuvres, allant à l'encontre de la politique et des médias où il existe une volonté de départager clairement la réalité de la fiction.

L'installation vidéographique de Clemencia Echeverri, intitulée Juegos de herencia, 2011, peut inviter à réfléchir sur cette problématique. L'œuvre est réalisée en Colombie, près du village d'El Valle dans la région du Pacifique nord. Elle a été filmée autour du 20 juillet, date qui marque la célébration de la Fête du Coq. L'installation vidéo est plutôt surprenante, huit écrans se font face et une dernière vidéo est projetée au sol sur un cercle de sable blanc. Cette dernière est l'espace du coq enterré, dont seule la tête dépasse du sol. Cette présence morte-vive, dans ce qui pourrait être une tombe improvisée de sable, impacte étrangement tout l'espace de l'œuvre. C'est aussi l'élément principal autour duquel s'organisent les événements de la fête. Sur les huit autres écrans, défilent au ralenti des images d'hommes, dans un temps presque suspendu, flottant. Un bruit de pluie inonde l'espace, puis des chants d'hommes accompagnés de tambours. Sur l'image, la tête d'un homme en gros plan à qui l'on bande les yeux; des bruits de foule, de sabre. D'autres hommes à qui l'on bande les yeux. Parfois, le coq prend l'espace de l'homme, il est là enterré. Même si l'image le montre en train de crier, l'espace est plein de silence. Ensuite, les images montrent l'excavation du trou, où se trouve sûrement enterré maintenant le coq. Puis des hommes reviennent de la mer. Les bruits de l'océan inondent l'espace. Enfin, à nouveau le coq, cette fois-ci pour le coup fatal. Rien n'est montré mais le bruit du sabre et le passage au noir de l'image laissent sous-entendre sa décapitation. Temporellement, les différentes actions sont dispersées, il n'y a pas de suite logique dans les actions, mais elles sont cependant liées par une logique narrative. Le spectateur est face à une histoire qui est racontée par bribes, par fragments. L'image aussi se défragmente souvent. Des ralentis à l'extrême s'apparentent à des photogrammes, des arrêts sur images alternent avec des vitesses de défilement normales. C'est là toute la richesse du langage cinématographique qui est mis au service de cette narration. L'histoire racontée est celle de cette fête, qui est un héritage espagnol que la communauté de ce village a assimilé et a fait sien au début du XX ${ }^{\text {ème }}$ siècle. Lors de cette fête, un coq est enterré vivant, des habitants de la communauté, les yeux bandés, doivent décapiter à la machette la tête de celui-ci.

L'œuvre de Clemencia Echeverri relate un fait réel, cependant, elle a recours à différents outils qui ne font pas partie de ce que l'on pourrait ranger sous l'étiquette du documentaire. Tout d'abord dans la forme de l'œuvre, dans le montage des images, dans la répétition des différents plans sur les différents écrans qui, tout en utilisant les ressources propres des arts visuels, s'éloignent des conventions propres au cinéma documentaire. L'œuvre n'est pas non plus le résultat d'un filmage en direct. L'artiste a recréé certaines actions pour faciliter leur filmage, la prise des détails, le positionnement de la caméra. Elle a donc recréé les gestes du rituel, dans le but de les adapter au langage cinématographique. 

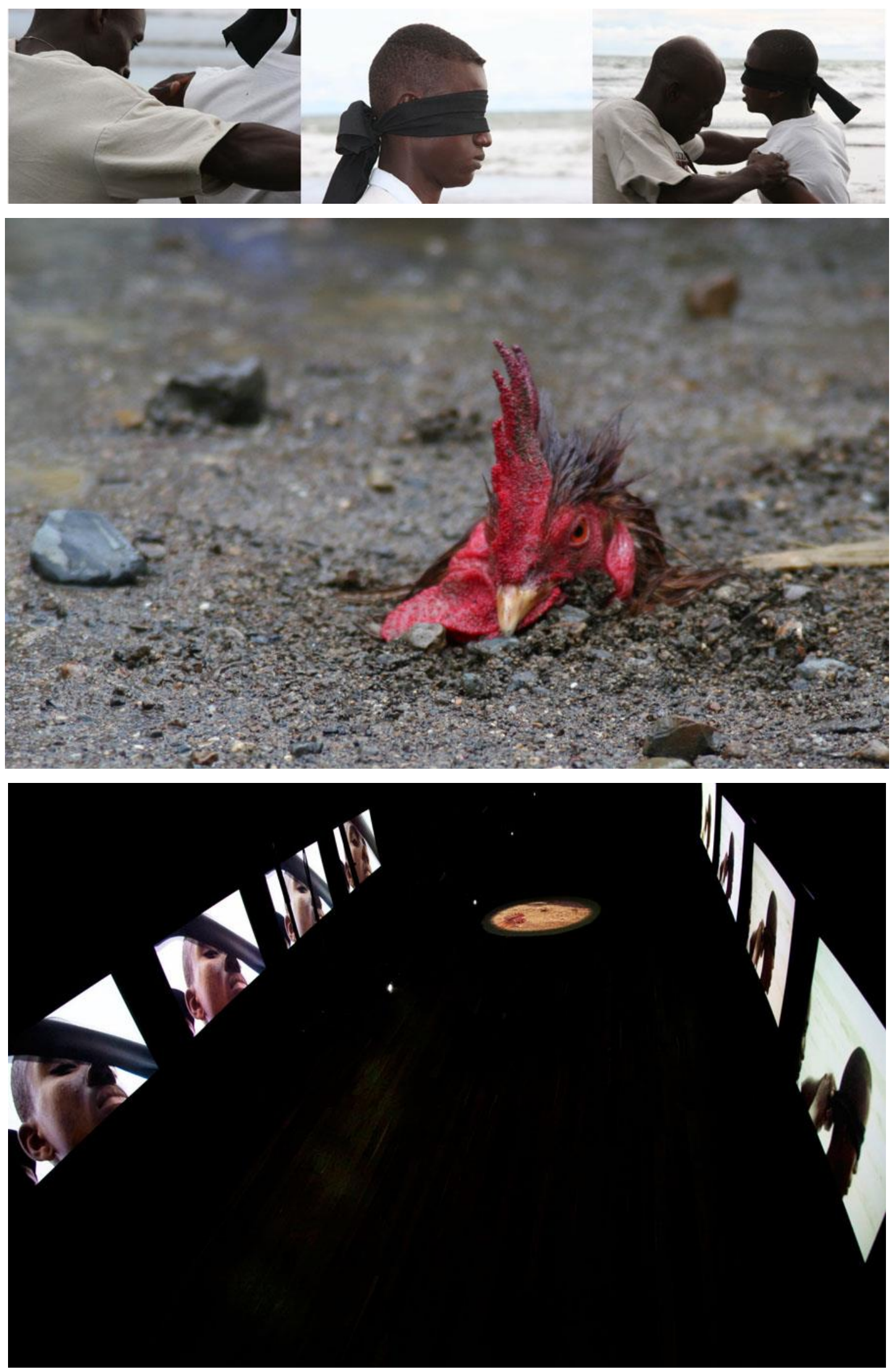

Echeverri Clemencia, Juegos de Herencia, (photogrammes et vue de l'installation), 24 minutes, huit projections latérales et une projection à terre, 2011, Bogota, Galeria Alonso Garcés. 
À propos de l'œuvre de Clemencia Echeverri, le philosophe colombien Gustavo Gomez écrit :

«Juegos de Herencia ouvre une réflexion sur ce que signifie faire l'histoire ou penser l'histoire, aspects qui sur ce point doivent être indiscernables. En effet, le rôle du son et de la vidéo, dans ce cas, sont utilisés pour révéler et intensifier le caractère violent qui en aucun cas ne pourrait se réduire à une seule image. $»^{5}$

Le philosophe pose sur le même plan de référence le fait de faire l'histoire et le fait de penser l'histoire par le biais d'une matière sensible qui est représentée ici par l'outil vidéographique. $\mathrm{Ne}$ s'agirait-il pas aussi de rendre l'histoire sensible ? De faciliter la compréhension de cette histoire, qui n'est pas uniquement transmise via le discours scientifique du chercheur, de l'historien, de l'anthropologue ; mais qui peut aussi être donnée à sentir.

La scénographie de l'œuvre Juegos de herencia, qui favorise l'immersion du spectateur dans une multitude d'écrans, d'images, exacerbe les sens, l'ouie, la vue, le toucher et l'odorat. C'est un flux sonore et visuel qui submerge le spectateur, favorisant les sensations corporelles et visuelles et ralentissant probablement les tentatives d'interprétation et de traduction de l'œuvre en mots et en pensées. L'installation ouvre à une expérience visuelle et auditive à la suite de laquelle pourra s'établir une lecture singulière de celle-ci. Sentir, faire l'expérience de l'histoire, de l'anthropologie pour la transmettre, la partager.

Dans son livre Cinéma / Paysages. Carnet de notes pour un film sur le Pô, Thierry Roche explore les territoires de la représentation du paysage entre le cinéma et l'anthropologie. Quand il s'agit de distinguer entre une approche scientifique et une approche artistique, il écrit :

«Il ne s'agit pas d'établir une relation étanche entre la poésie et la science, entre la science et l'humain - comme tend à le faire Flaherty - mais de penser le monde et l'humanité de manière conjointe à partir d'approches scientifiques et artistiques, les unes et les autres devant se nourrir mutuellement. L'humain est l'objet et le sujet de la science et de l'art. »

Clemencia Echeverri ne donne pas d'informations qui nous permettraient de contextualiser la fonction de cette tradition dans la communauté, d'en comprendre ses enjeux, ses héritages ; parce que son intérêt est ailleurs. Comme l'écrit Thierry Roche, son attention se situe au cœur de l'humain. Au-delà du rituel, c'est la relation de l'homme avec le monde, avec le passé, avec la violence, avec le monde animal qui se manifeste dans cette œuvre. Cette œuvre traverse les disciplines, elle s'en nourrit, elle ne les met pas en compétition. Clemencia Echeverri pense le monde à travers différentes approches qui nourrissent le processus de recherche-création. Je soutiendrais qu'un art anthropologique est une construction fictionnelle dans laquelle se trouve mêlée l'expérience de l'artiste à l'autre, humain ou non-humain, mais aussi à l'autre discipline.

Mes explorations plastiques s'initient majoritairement dans ces territoires de l'hybridation, dans ces rencontres singulières qui bouleversent et qui donnent vie à d'étranges fictions. Elles construisent des mondes (kosmos) plastiques, sensibles, au seuil des territoires, des règnes animaux, végétaux, minéraux et humains. Elles se nourrissent des savoir-faire, des mythes, des histoires qui Now, 2013, p. 40. 
rendent le monde vivant et qui nous font appartenir au monde. Elles tentent à leur échelle, de « recosmiser » le monde, sans doute et avant tout en rappelant que l'homme est vivant, comme tout ce qui l'entoure, que toutes les entités qui l'habitent sont douées de vie et qu'elles méritent de ce fait le respect. 